\title{
Das Wasser ist abgelassen, es sprudeln die Worte
}

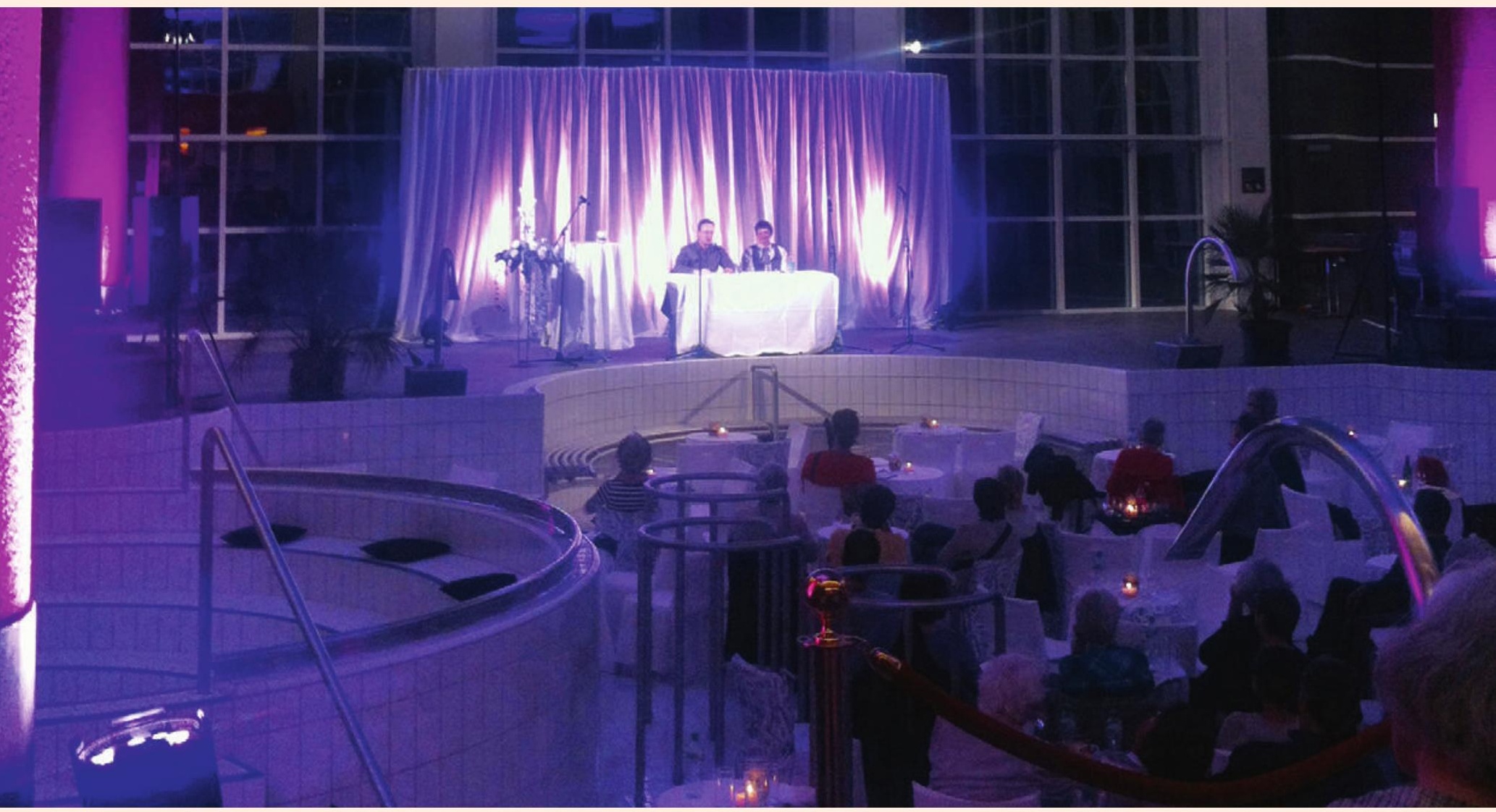

Die Alpentherme in Leukerbad einmal anders: Nicht Badende, sondern Zuhörende teilen sich das Schwimmbecken und lauschen Christoph Simon.

Nadja Pecinska

npecinska[at]emh.ch
Langsam kurvt der Bus hinauf in die Höhe, von Leuk im Walliser Rhonetal nach Leukerbad, wohin bereits 1779 ein Deutscher namens Goethe die internationale Literatur gebracht hat. «Wir waren nun schon drei Stunden aufwärts in das ungeheure Gebirg gestiegen, das Wallis von Bern trennt. Es ist eben der Stock von Bergen, der in einem fort vom Genfer See bis auf den Gotthard läuft und auf dem sich in dem Berner Gebiet die großen Eis- und Schneemassen eingenistet haben. Hier sind oben und unten relative Worte des Augenblicks. Ich sage: «Unter mir auf einer Fläche liegt ein Dorf, und eben diese Fläche liegt vielleicht wieder an einem Abgrund, der viel höher ist als mein Verhältnis zu ihr» [1]. So beschrieb Goethe seinen ersten Eindruck von Leukerbad. 1951 überwinterte ein gewisser James Baldwin im Dorf unter der Gemmi. Als schwarzer Homosexueller wurde er nicht gerade mit offenen Armen empfangen. Über diese Erfahrung und den ungleichen Blick, mit dem Schwarze und Weisse einander wahrnehmen, hat James Baldwin einen Essay verfasst, eine
Analyse von Rassismus, die wohl heute wieder eine traurige Aktualität erlangt [2]. Seit 19 Jahren sorgt nun das Internationale Literaturfestival Leukerbad dafür, dass diese Tradition internationaler Schriftsteller in Leukerbad nicht abbricht. Jeweils für ein Wochenende im Juli schlängeln sich nicht nur Badegäste, Skifahrer und Wandervögel die einzige Strasse hinauf, die das Bergdorf mit dem Rest der Welt verbindet - in alle anderen Richtungen müssen historische Alpenpässe überquert werden -, sondern auch international gefeierte Autorinnen und Autoren wie Ilja Trojanow, Christoph Heim, Herta Müller, Jonathan Safran Foer oder Connie Palmen fanden in den letzten Jahren ihren Weg nach Leukerbad. Natürlich waren über die Jahre auch zahlreiche Schweizer Stimmen wie die von Lukas Bärfuss, Peter Stamm, Rolf Lappert, Roger Monnerat oder Pedro Lenz zu hören.

Wolkenverhangen zeigten sich am ersten Tag des Festivals die Felswände, die Leukerbad für die einen bedrohlich, für die anderen beschützend umschliessen. Das verhiess nichts Gutes für die jedes Jahr statt- 
findende Mitternachtslesung auf der Gemmi. Für diesen Anlass nehmen die Gemmi-Bahnen jeweils um 23 Uhr ihren Betrieb nochmals auf und fahren die abenteuerlustigen und nimmersatten Festivalbesucher über den schwarzen Abgrund auf 2350 Meter, wo zur Geisterstunde eine Lesung der ganz besonderen Art stattfindet. Und tatsächlich, Wind und Sturm waren für die Nacht angesagt; der literarische schweizerisch-österreichische Schlagabtausch zwischen Christoph Simon und Händl Klaus musste sozusagen von der Luft ins Wasser verlegt werden. Bis am Vortag sprudelten im Schwimmbecken der Walliser Alpentherme Warmwasserdüsen, die Besucher waren mit Badehose und Badetuch ausgestattet. Nun fanden weissgedeckte Tische im Schwimmbecken Platz, an denen gutgekleidete Gäste bei Wein und Walliser Teller den vom Beckenrand rezitierenden Autoren lauschten. Und Christoph Simon zu lauschen lohnte sich in der Tat - nicht nur, wenn er, stehend, mit eleganter Weste und den Händen in den Hosentaschen, von einer Vasektomie-Erfahrung erzählt (s. Kasten).

Vor diesem Höhepunkt, der also doch nicht in der Höhe stattfinden konnte, gehörte der Abend in der Alpentherme drei fremdsprachigen Autorinnen. Den Auftakt machte die Türkin Sema Kaygusuz, es folgte die australische Autorin Gail Jones und den Schlussstrich setzte Maaza Mengiste aus Äthiopien. Schade, dass es sich bei den drei Lesungen wortwörtlich um Lesungen handelte. Nur kurz lasen die Autorinnen jeweils in ihrer Sprache, dann kam die deutsche Stimme zu Wort (Ulrike Arnold, Michaela Wendt), die die verbleibende halbe Stunde die deutsche Übersetzung erklingen liess. Lesen können die Anwesenden alle selbst und sollten das auch tun, denn am Ende des Tages wollen die Autoren, dass ihre Bücher gekauft und gelesen werden. Viel interessanter wäre es, etwas über die Autoren zu erfahren, ihr Leben, ihr Wirken, ihr Ringen um die Worte.

Solche Gespräche mit Autoren fanden zu spärlich statt. Einer dieser raren Momente war das literarische Horsd'œuvre zwischen Stefan Zweifel und Navid Kermani, die sich über eine Liebe der Grösse eines kleinen Spucknapfs unterhielten (Navid Kermani, Große Liebe. Hanser Verlag, München 2014). Aber auch mit honorierten Gästen schmückte sich das Literaturfestival. Anwesend mit ihrem Ungeheuer die letztjährige Gewinnerin des Deutschen Buchpreises Terézia Mora (Terézia Mora, Das Ungeheuer. Luchterhand Literaturverlag, München 2013) und der Gewinner des Schweizer Buchpreises Jens Steiner (Jens Steiner, Carambole. Dörlemann Verlag, Zürich 2013).

Ein literarischer Leckerbissen war die Lesung von Thomas Kapielski im alten Bahnhof. Er erzählte von Schafen, «die auf Wolle hochgezüchteten Viecher», die sich im nassen Monat April derart mit Wasser vollsaugen, dass sie «im Wind umkippen und nicht mehr von alleine hochkommen». Da ist der Dorftrottel
Christoph Simon über eine Vasektomie-Erfahrung, vorgetragen am Literaturfestival Leukerbad

Ist es nicht schön, einmal ins Spital zu gehen, nicht weil man muss, sondern weil man will? So eine Vasektomie ist für uns Männer dasselbe wie eine Schönheitsoperation für Frauen in der Menopause,

etwas, das wir uns einfach mal gönnen wollen und für uns selbst tun.

Ich frage die Urologin, ob es mir erlaubt sei, mich in der Hundestellung operieren zu lassen. Mir scheint, die Hundestellung sei sowohl die gemütlichste Geburtsstellung als auch die ideale Defertilisationsposition.

Sie erlaubt es nicht, und aufgestützt auf die Ellenbogen verfolge ich mit, was sie am Sitz meiner Seele macht.

Das Skalpell legt den Samenleiter frei und mir wird etwas anders,

ich betrachte den unorganisierten Kandinsky an der Wand, das Stillleben aus Siemens-Computer,

Tritel-Telefon und Infusionsständer, und als ich mich wieder dem Geschehen zuwende, wird gerade etwas verschweisst.

Zwischen meinen Beinen steigen feine Rauchzeichen auf und es riecht leicht verbrannt.

Aha, denke ich, das ist nun meine Fruchtbarkeit, die mit dem Geruch einer Fliege, die in der Halogenlampe verglüht, engelsgleich entschwebt.

Meine Herren im zeugungsfähigen Alter: Ein paar Tage nach einer Vasektomie können Sie wieder ohne Kissen zwischen den Schenkeln schlafen, das Veilchenblaugelbgrünviolett klingt $a b$, und Sie spüren, wie Ihr Leben viel wirklicher und intensiver ist als es vorher. Das Gras grüner, der Holunder weisser, die Sonne heller. Ihre Gefährtinnen spüren Ihre neue Zugänglichkeit und geniessen Ihre entspannte Art, der Zukunft entgegen zu schreiten. Und am Abend machen Sie sich vielleicht gegenseitig drei grosse Zahnpastapunkte und drei kleine Zahnpastapunkte auf die Zahnbürste, was heisst: Fruchtbarkeit ist heilbar.

(Abdruck mit freundlicher Genehmigung von Christoph Simon. www.christophsimon.ch. Seine Bücher sind im bilgerverlag erschienen. www.bilgerverlag.ch)

gefragt, dessen Rolle, wie der Autor berichtet, er damals einnehmen musste, um die Viecher wieder auf die Beine zu stellen. «Dafür kriegst du einen derart dankbaren Blick, so voller Einfalt, aber mit der englischsten Engel keuschester Anmut, dass es dich schier hinhaut!» Auf Kapielskis ersten Roman (er hat bisher nie Romane geschrieben, da ihn Sätze wie «er 


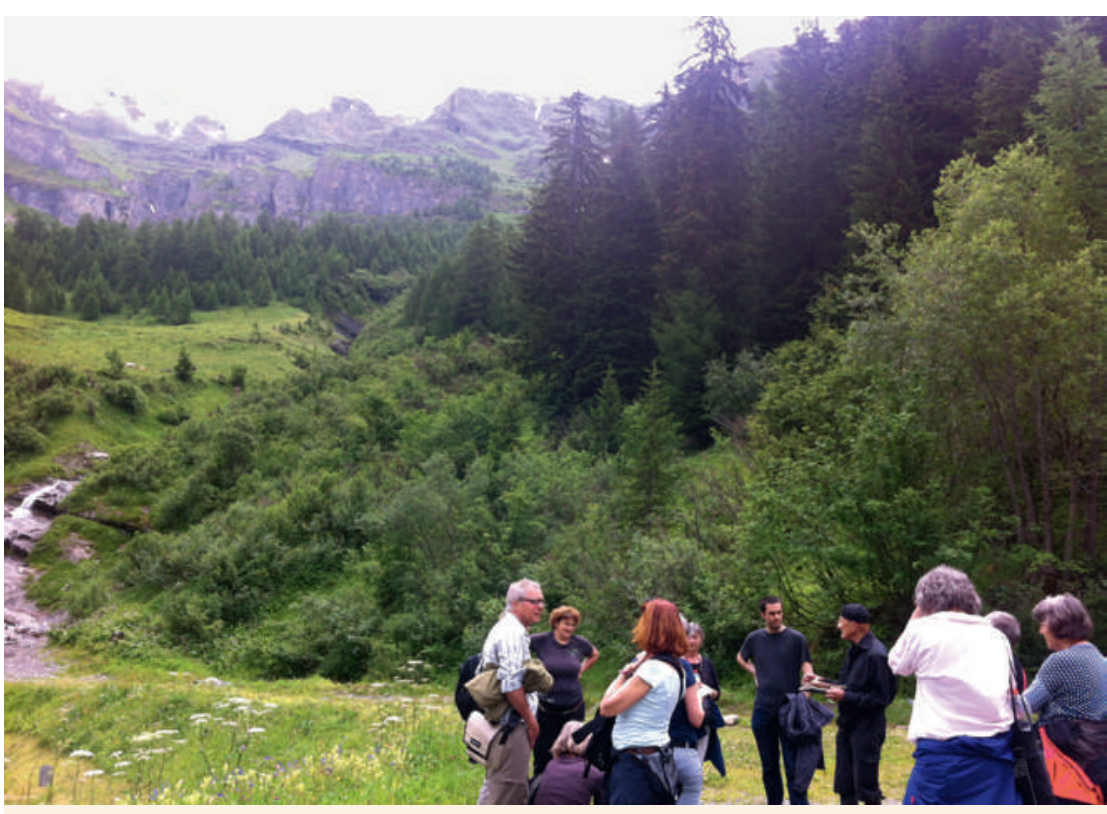

Literatur mitten in den Bergen: Bodo Hell rezitiert während des Dala-Schlucht-Spaziergangs seine Gedichte.

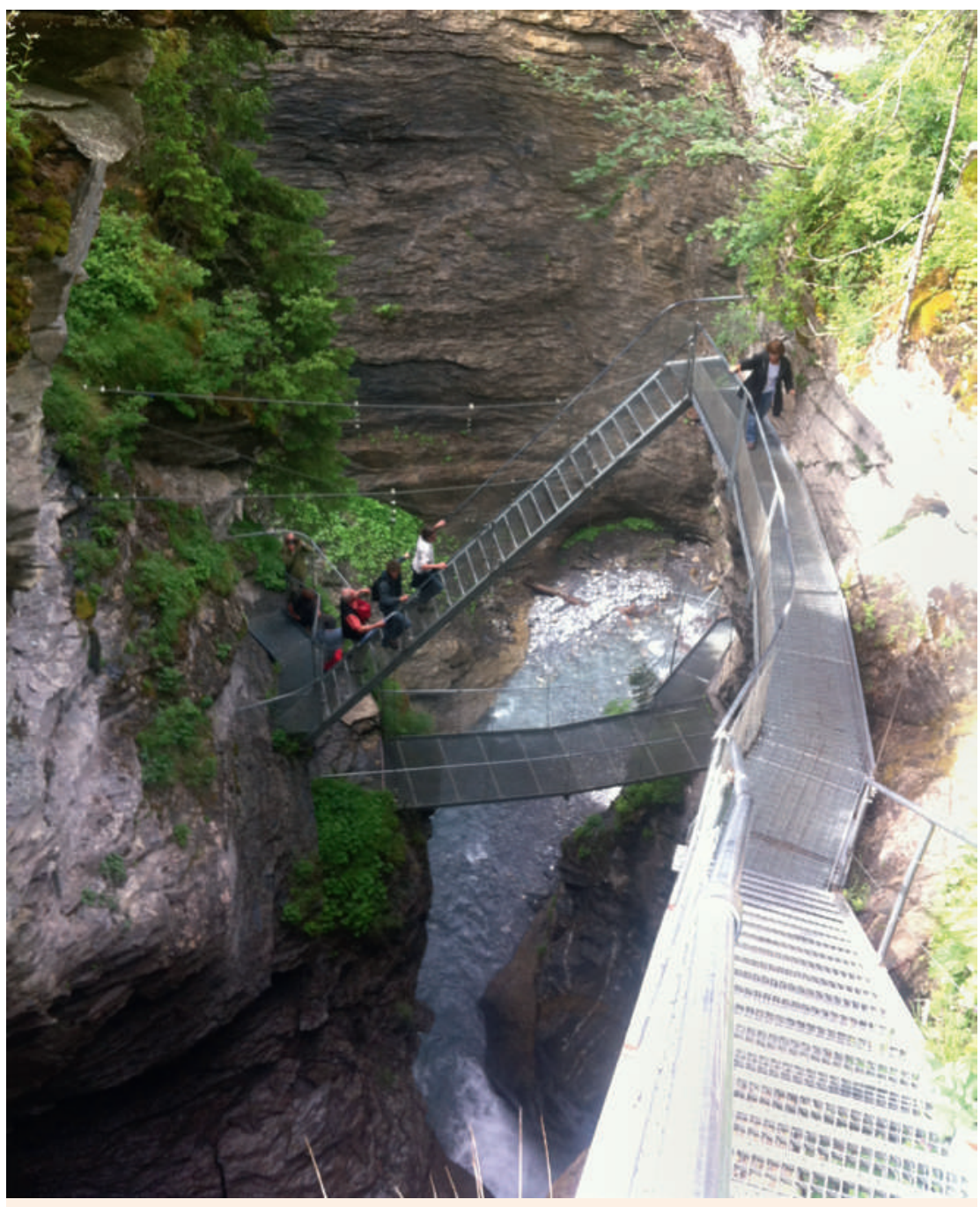

Die Dala-Schlucht: nichts für schwache Nerven. sass auf der Bettkante und rauchte eine Zigarette» masslos ärgern) dürfen wir uns freuen, er trägt den Titel Je dickens, destojewski! und soll Ende August 2014 bei Suhrkamp erscheinen.

Ein Programmpunkt durfte auch in diesem Jahr nicht fehlen: der literarische Dala-Schlucht-Spaziergang, dem der österreichische Autor Bodo Hell mit seinen Alltagswahnsinn-Wort-Aneinanderreihungen den Rahmen gab. Und dieser Rahmen bzw. ein Geländer war für den einen oder anderen Mitspaziergänger enorm wichtig, führt dieser Schluchtweg doch über Gitterstege, in den Felsen eingeschraubte Leitern und schwindelerregende Hängebrücken. Bodo Hell ist diese Abgründe gewohnt, weilt er doch seit über 30 Jahren jeweils im Sommer am Dachstein in der Steiermark, wo er Rinder und Ziegen betreut, über die ein Film entstanden ist, der am Festival zu sehen war (Im Augenblick. Die Historie und das Offene. Regie: Angela Summereder, Othmar Schmiderer, Österreich 2013).

Der Samstagabend gehörte einerseits einem «Abend für Clarice», der der verstorbenen brasilianischen Schriftstellerin Clarice Lispector gewidmet wurde, sowie dem «Literarischen Abend» in der Alpentherme. Hintereinander in kurzen Blöcken lasen Autoren wie Tanja Maljartschuk, Roman Graf, Katharina Faber, Raphael Urweider, Antonio Fian und auch nochmals Gail Jones, Bodo Hell, Thomas Kapielski und Christoph Simon kurze und kurzweilige Texte. Ein wahres Wortfest bescherte den Badegästen im Trockenen Saša Stanišic, der diesjährige Gewinner des Preises der Leipziger Buchmesse, mit dem Einstieg in seine Geschichte: «Meine Mu wiegt doppelt so viel wie mein Pa. Sie wiegt 130 Kilo. Im Frühling kommen 30 Kilo schwere Gedanken dazu (Sorgen, Ängste, Scham, generelle Lustlosigkeit). Dann legt sich meine 160-Kilo-Mu in die Narzissen im Garten, weil im Liegen die dunklen Wolken circa hundertsechzig Zentimeter weiter weg sind» (Saša Stanišic: Vor dem Fest. Roman. Luchterhand Verlag, München 2014). Insgesamt wurde das Publikum in den drei Tagen von 30 Autorinnen und Autoren mit Wörtern, Sätzen, Poesie und Geschichten beschenkt.

«Glück ist, wenn man mehr aus dem Leben herausholt, als das Schicksal für einen vorgesehen hat» (Christoph Simon). Sollte das Schicksal Sie bislang noch nicht ans Literaturfestival Leukerbad gebracht haben, dann fahren Sie hin. Das nächste und sogleich auch 20-jährige Jubiläums-Festival findet vom 3. bis 5. Juli 2015 statt.

\section{Literatur}

1 Briefe aus der Schweiz, 2. Abteilung [zur 2. Reise in die Schweiz 1779]. Berliner Ausgabe, Bd. 15. Briefe aus der Schweiz. Zweite Abteilung. Berlin/ Weimar: Aufbau Verlag, S. 7-63.

2 Baldwin J. Fremder im Dorf. Ein schwarzer New Yorker in Leukerbad. Edition sacré. 2. Aufl.; 2012. 